\title{
THE ESSENTIAL SPECTRUM OF A PERTURBED OPERATOR ARISING IN TWO-DIMENSIONAL MAGNETOHYDRODYNAMICS
}

\author{
M. FAIERMAN ${ }^{\otimes}$ and R. MENNICKEN \\ (Received 17 March 2009; accepted 21 December 2009) \\ Communicated by J. J. Koliha
}

\begin{abstract}
Descloux and Geymonat considered a model problem in two-dimensional magnetohydrodynamics and conjectured that the essential spectrum has an explicitly given band structure. This conjecture was recently proved by Faierman, Mennicken, and Möller by reducing the problem to that for a $2 \times 2$ block operator matrix. In a subsequent paper Faierman and Mennicken investigated the essential spectrum for the problem arising from a particular type of perturbation of precisely one of the operator entries in the matrix representation cited above of the original problem considered by Descloux and Geymonat. In this paper we extend the results of that work by investigating the essential spectrum for the problem arising from particular types of perturbations of all but one of the aforementioned operators. It remains an open question whether one can perturb the exceptional operator in such a way as to leave the essential spectrum unchanged.
\end{abstract}

2000 Mathematics subject classification: primary 35H99; secondary 47A10.

Keywords and phrases: essential spectrum, perturbed operator, magnetohydrodynamics.

\section{Introduction}

Descloux and Geymonat [DG1, DG2] considered a model problem in two-dimensional magnetohydrodynamics and a conjecture was made concerning the structure of the essential spectrum. By approaching this problem from an operator-theoretic point of view and by exploiting the fact that the coefficients of this operator depended only upon one of the space variables concerned, Faierman et al. [FMM] were able, with the use of Fourier series expansions and some results of [GK] concerning systems of integral equations, to completely prove the conjecture. In a subsequent paper Faierman and Mennicken [FM] considered a perturbation of the above operator by the addition of a particular type of potential, so that the coefficients of the perturbed operator no longer depended only upon one of the space variables concerned, and the essential

(C) 2010 Australian Mathematical Publishing Association Inc. 1446-7887/2010 \$16.00 
spectrum of the perturbed operator was again completely determined. In this paper we extend the work of [FM] by considering a perturbation of the original operator by a potential of a much more general type than hitherto considered, and then investigate the essential spectrum of this perturbed operator.

To explain our work in more detail, let $\Omega$ denote the region in the $(s, t)$-plane defined by the inequalities $0<s<1,0<t<2 \pi$, and let $\partial_{1}=\partial / \partial s, \partial_{2}=\partial / \partial t$. Then motivated by the fact that we have to deal with spaces of functions which are periodic with respect to the variable $t$ of period $2 \pi$, let us introduce the function space $C_{0, \pi}^{\infty}(\Omega)$ defined as the set of all $f \in C^{\infty}(\bar{\Omega})$ with support contained in $[\epsilon, 1-\epsilon] \times[0,2 \pi]$ for some $\epsilon$ in $(0,1 / 2)$ (which may depend on $f$ ) such that $\partial_{2}^{j} f(\cdot, 0)=\partial_{2}^{j} f(\cdot, 2 \pi)$ for all $j \geq 0$, where supp denotes support and $C^{\infty}(\bar{\Omega})=\left.C^{\infty}\left(\mathbb{R}^{2}\right)\right|_{\Omega}$. It is clear that $C_{0, \pi}^{\infty}(\Omega)$ is a dense subspace of

$$
L_{2}\left(\Omega, s^{-1}\right)=\left\{\left.f \in L_{2}(\Omega)\left|\int_{\Omega} s^{-1}\right| f\right|^{2} d x<\infty\right\},
$$

where $d x$ denotes the element of two-dimensional Lebesgue measure. Then with $u=$ $\left(u_{1}, u_{2}\right)^{T}, v=\left(v_{1}, v_{2}\right)^{T}$ (where ${ }^{T}$ denotes the transpose) denoting vector functions in $C_{0, \pi}^{\infty}(\Omega)^{2}$, let us introduce the sesquilinear form on $L_{2}\left(\Omega, s^{-1}\right)^{2}$,

$$
\begin{gathered}
\mathbf{l}(u, v)=\int_{\Omega} \frac{1}{s}\left[\left(\omega^{-1} \partial_{1} u_{1}+s^{-1} \partial_{2} u_{2}\right) \overline{\left(\omega^{-1} \partial_{1} v_{1}+s^{-1} \partial_{2} v_{2}\right)}\right. \\
\left.+\omega^{-2} \partial_{2} u_{1} \overline{\partial_{2} v_{1}}+\partial_{2} u_{2} \overline{\partial_{2} v_{2}}\right] d x
\end{gathered}
$$

and the associated operator with domain $C_{0, \pi}^{\infty}(\Omega)^{2}$,

$$
\mathbf{L}_{0}=\left(\begin{array}{cc}
A & B \\
C & D_{0}
\end{array}\right)
$$

where $\omega$ is a positive constant,

$$
\begin{gathered}
A=-\omega^{-2} s \partial_{1} \frac{1}{s} \partial_{1}-\omega^{-2} \partial_{2}^{2}, \quad B=-s \partial_{1} \frac{\omega^{-1}}{s^{2}} \partial_{2}, \\
C=-\frac{\omega^{-1}}{s} \partial_{2} \partial_{1}, \quad D_{0}=-\frac{1+s^{2}}{s^{2}} \partial_{2}^{2} .
\end{gathered}
$$

In [FMM] it is shown that the form $\mathbf{l}$ is symmetric, nonnegative, and closable in $L_{2}\left(\Omega, s^{-1}\right)^{2}$; and the nonnegative, selfadjoint operator associated with its closure, that is, Friedrich's extension of $\mathbf{L}_{0}$, is denoted by $\mathbf{L}_{F}$ (see [K, Theorem 2.1, p. 322]). Then the main result of [FMM] was the complete characterization of $\sigma_{\mathrm{ess}}\left(\mathbf{L}_{F}\right)$, the essential spectrum of $\mathbf{L}_{F}$, which proved the conjecture of Descloux and Geymonat cited above.

In $[\mathrm{FM}]$ a perturbation of the above problem was considered by replacing the sesquilinear form (1.1) by the form

$$
\mathbf{l}^{(1)}(u, v)=\mathbf{l}(u, v)+\int_{\Omega} \frac{1}{S} a(\cdot, \cdot) u_{1} \overline{v_{1}} d x,
$$


where $a \in L_{\infty}(\Omega)$ and is real-valued. The operator associated with $\mathbf{l}^{(1)}$ is then $\mathbf{L}_{0}^{(1)}$, obtained from the operator $\mathbf{L}_{0}$ of (1.2) by replacing $A$ there by $A^{(1)}=A+S_{a}$, where for any measurable function $g$ defined on $\Omega$, we use the notation $S_{g}$ to denote the operator of multiplication in $L_{2}\left(\Omega, s^{-1}\right)$ induced by $g$. It is shown in [FM] that the sesquilinear form $\mathbf{l}^{(1)}$ is densely defined, symmetric, and closable in $L_{2}\left(\Omega, s^{-1}\right)^{2}$, and with $\mathbf{L}_{F}^{(1)}$ denoting the selfadjoint operator associated with its closure, the main result of that paper is the proof that $\sigma_{\text {ess }}\left(\mathbf{L}_{F}^{(1)}\right)=\sigma_{\text {ess }}\left(\mathbf{L}_{F}\right)$ under certain assumptions concerning $a$.

In this paper we extend the work of [FM] by considering a more general type of perturbation than considered there. Namely, we replace the sesquilinear form (1.3) by the form

$$
\begin{gathered}
\mathbf{l}^{(2)}(u, v)=\mathbf{l}_{1}(u, v)+\int_{\Omega} \frac{1}{s} b(\cdot, \cdot) u_{2} \bar{v}_{1} d x+\int_{\Omega} \frac{1}{s} c(\cdot, \cdot) u_{1} \bar{v}_{2} d x \\
+\int_{\Omega} \frac{1}{s} d(\cdot, \cdot) u_{2} \bar{v}_{2} d x
\end{gathered}
$$

where $b, c$, and $d$ are functions in $L_{\infty}(\Omega), b=\bar{c}$, and $d$ is real-valued. The operator associated with $\mathbf{I}^{(2)}$ is then $\mathbf{L}_{0}^{(2)}$, obtained from the operator $\mathbf{L}_{0}$ of (1.2) by replacing the operators $A, B, C$, and $D_{0}$ there by the operators $A^{(1)}, B^{(1)}, C^{(1)}$, and $D_{0}^{(1)}$, respectively, where $B^{(1)}=B+S_{b}, C^{(1)}=C+S_{c}$, and $D_{0}^{(1)}=D_{0}+S_{d}$. As we shall see, the form $\mathbf{I}^{(2)}$ is densely defined, symmetric, and closable in $L_{2}\left(\Omega, s^{-1}\right)^{2}$, and we shall denote the selfadjoint operator associated with its closure by $\mathbf{L}_{F}^{(2)}$. Then the object of this paper is to prove that $\sigma_{\mathrm{ess}}\left(\mathbf{L}_{F}^{(2)}\right)=\sigma_{\mathrm{ess}}\left(\mathbf{L}_{F}\right)$ under certain conditions concerning the potentials $a, b$, and $d$ (see Theorem 3.1 below).

In Section 2 some necessary results are gathered together from both [FM, FMM], and these are then used in Section 3 to prove our main result, Theorem 3.1.

\section{Preliminaries}

In this section we gather together some results from [FMM] which we require for our purposes; and to this end we need the following notation. For a measurable subset $G$ of $\Omega$ or of the interval $0<s<1$, we denote by $L_{2}\left(G, s^{-1}\right)$ the weighted Lebesgue space relative to $s^{-1}$ times Lebesgue measure in the Euclidean space containing $G$, and denote by $(\cdot, \cdot)_{0, G, s^{-1}}$ and $\|\cdot\|_{0, G, s^{-1}}$ the inner product and norm in $L_{2}(G, 1 / s)$. For a nonnegative integer $m$ we let $H^{m}(G)$ and $H^{m}((0,2 \pi))$ denote the usual Sobolev spaces of order $m$ related to $L_{2}(G)$ and $L_{2}((0,2 \pi))$, and let

$$
H^{m}\left(\Omega, s^{-1}\right)=\left\{f \in H^{m}(\Omega) \mid \partial^{\alpha} f \in L_{2}\left(\Omega, s^{-1}\right) \text { for }|\alpha| \leq m\right\},
$$

where $\alpha=\left(\alpha_{1}, \alpha_{2}\right)$ is a multi-index whose length $\alpha_{1}+\alpha_{2}$ is denoted by $|\alpha|$, and $\partial^{\alpha}=\partial_{1}^{\alpha_{1}} \partial_{2}^{\alpha_{2}}$. We also equip $H^{m}\left(\Omega, s^{-1}\right)$ with the inner product 


$$
(f, g)_{m, \Omega, s^{-1}}=\sum_{|\alpha| \leq m} \int_{\Omega} s^{-1} \partial^{\alpha} f \overline{\partial^{\alpha} g} d x
$$

and norm $\|f\|_{m, \Omega, s^{-1}}=(f, f)_{m, \Omega, s^{-1}}^{1 / 2}$, where $f$ and $g$ denote vectors in this space. Furthermore, we introduce the spaces

$$
\begin{aligned}
& H_{\pi}^{m}\left(\Omega, s^{-1}\right) \\
& =\left\{f \in H^{m}\left(\Omega, s^{-1}\right) \mid \partial_{2}^{j} f(\cdot, 0)=\partial_{2}^{j} f(\cdot, 2 \pi) \text { for } j=0, \ldots, m-1\right\}, \\
& Z_{\pi}^{m}\left(\Omega, s^{-1}\right) \\
& =\left\{f \in L_{2}\left(\Omega, s^{-1}\right) \mid s^{-j} \partial_{2}^{j} f \in L_{2}\left(\Omega, s^{-1}\right) \text { for } j=0, \ldots, m,\right. \\
& \left.\partial_{2}^{j} f(\cdot, 0)=\partial_{2}^{j} f(\cdot, 2 \pi) \text { for } j=0, \ldots, m-1\right\}, \\
& H_{\pi}^{m}((0,2 \pi)) \\
& =\left\{f \in H^{m}((0,2 \pi)) \mid \partial_{2}^{j} f(0)=\partial_{2}^{j}(2 \pi) \text { for } j=0, \ldots, m-1\right\}, \\
& H^{m}\left((0,1), s^{-1}\right) \\
& =\left\{f \in L_{2}\left((0,1), s^{-1}\right) \mid \partial_{1}^{j} f \in L_{2}\left((0,1), s^{-1}\right) \text { for } j=1, \ldots, m\right\},
\end{aligned}
$$

where $H^{m}(G)$, (and hence $\left.H_{\pi}^{m}(\Omega)\right)$ as well as $H^{m}((0,2 \pi))$ (and hence $H_{\pi}^{m}((0,2 \pi))$ ) are equipped with their standard inner products and norms, $H^{m}\left((0,1), s^{-1}\right)$ is equipped with the inner product

$$
(f, g)_{m,(0,1), s^{-1}}=\sum_{j=0}^{m} \int_{0}^{1} s^{-1} \partial_{1}^{j} f \overline{\partial_{1}^{j} g} d s
$$

and norm

$$
\|f\|_{m,(0,1), s^{-1}}=(f, f)_{m,(0,1), s^{-1}}^{1 / 2},
$$

where $f$ and $g$ denote vectors in this space, while we equip $Z_{\pi}^{m}\left(\Omega, s^{-1}\right)$ with the inner product

$$
(\cdot, \cdot)_{Z_{\pi}^{m}\left(\Omega, s^{-1}\right)}=\sum_{j=0}^{m}\left(s^{-j} \partial_{2} \cdot, s^{-j} \partial_{2} \cdot\right)_{0, \Omega, s^{-1}}
$$

and norm

$$
\|\cdot\|_{Z_{\pi}^{m}\left(\Omega, s^{-1}\right)}=(\cdot, \cdot)_{Z_{\pi}^{m}\left(\Omega, s^{-1}\right)}^{1 / 2} .
$$

It is not difficult to deduce from the results of [FMM] (see in particular Proposition 4.3 there) that $C_{0, \pi}^{\infty}(\Omega)$ is dense in $L_{2}\left(\Omega, s^{-1}\right)$ and in $Z_{\pi}^{m}\left(\Omega, s^{-1}\right)$ for $m=1,2$.

In Section 1 we introduced the functions $a, b, c$ and $d$ and indicated some of their properties. We now list some further properties which we assume these functions to satisfy. To this end we require the following definition.

DEFINITION 2.1. We say that the function $f$ satisfies condition (A) if:

(1) $f \in L_{\infty}(\Omega)$ such that $f(s, \cdot) \in H_{\pi}^{2}((0,2 \pi))$ for almost every $s$ in $0<s<1$ and $\partial_{2}^{2} f \in L_{\infty}(\Omega)$ 
(2) if $0<\delta<1$ and

$$
\rho_{f}(\delta)=\left\|\left(\int_{0}^{2 \pi}\left(|f(s, \cdot)|^{2}+\left|\partial_{2}^{2} f(s, \cdot)\right|^{2}\right) d t\right)^{1 / 2}\right\|_{L_{\infty}((0, \delta))},
$$

then $\rho_{f}(\delta) \rightarrow 0$ as $\delta \rightarrow 0$.

ASSUMPTION 2.2. We henceforth suppose that $a,|b|^{2}$, and $d^{2}$ satisfy condition (A).

We stated in Section 1 that the form $\mathbf{I}$ with domain $C_{0, \pi}^{\infty}(\Omega)^{2}$ (see (1.1)) is symmetric, nonnegative, and closable in $L_{2}\left(\Omega, s^{-1}\right)^{2}$, and we let $\tilde{\mathbf{l}}$ denote its closure. Also it was shown in [FM] that the perturbed form $\mathbf{l}^{(1)}$, with domain $C_{0, \pi}^{\infty}(\Omega)^{2}$, is symmetric, closable in $L_{2}\left(\Omega, s^{-1}\right)^{2}$, and has lower bound greater than or equal to $-\gamma_{a}$, where $\gamma_{a}=\|a\|_{L_{\infty}(\Omega)}$. Furthermore, if $\tilde{\mathbf{l}}^{(1)}$ denotes its closure, then $\tilde{\mathbf{l}}$ and $\tilde{\mathbf{l}}^{(1)}$ have the same domain and on their common domain, $\tilde{\mathbf{l}}^{(1)}(\cdot, \cdot)=\tilde{\mathbf{l}}(\cdot, \cdot)+\left(S^{(1)} \cdot, \cdot\right)_{L_{2}\left(\Omega, s^{-1}\right)^{2}}$, where $S^{(1)}$ denotes the $2 \times 2$ matrix operator whose entry in the first row and first column is $S_{a}$ and all other entries are 0 . In addition, if $\mathbf{L}_{F}^{(1)}$ denotes the selfadjoint operator associated with the form $\tilde{\mathbf{I}}^{(1)}$, then $\mathbf{L}_{F}$ and $\mathbf{L}_{F}^{(1)}$ have the same domain and on their common domain, $\mathbf{L}_{F}^{(1)}=\mathbf{L}_{F}+S^{(1)}$. Finally, if we let $\gamma_{0}=2\left(\gamma_{a}+\gamma_{b}+\gamma_{d}\right)$, where $\gamma_{b}=\|b\|_{L_{\infty}(\Omega)}$ and $\gamma_{d}=\|d\|_{L_{\infty}(\Omega)}$, and refer again to Section 1 for notation, then we can argue with the form $\mathbf{I}^{(2)}$ as we argued with the form $\mathbf{l}^{(1)}$ in [FM] to obtain the following result.

Proposition 2.3. The perturbed form $\mathbf{I}^{(2)}$, with domain $C_{0, \pi}^{\infty}(\Omega)^{2}$, is symmetric, closable in $L_{2}\left(\Omega, s^{-1}\right)^{2}$, and has lower bound at least $-\gamma_{0}$. Furthermore, if $\tilde{\mathbf{I}}^{(2)}$ denotes its closure, then $\tilde{\mathbf{I}}$ and $\tilde{\mathbf{I}}^{(2)}$ have the same domain and on their common domain, $\tilde{\mathbf{l}}^{(2)}(\cdot, \cdot)=\tilde{\mathbf{l}}(\cdot, \cdot)+\left(S^{(2)} \cdot, \cdot\right)_{L_{2}\left(\Omega, s^{-1}\right)^{2}}$, where

$$
S^{(2)}=\left(\begin{array}{ll}
S_{a} & S_{b} \\
S_{c} & S_{d}
\end{array}\right)
$$

Finally, if $\mathbf{L}_{F}^{(2)}$ denotes the selfadjoint operator in $L_{2}\left(\Omega, s^{-1}\right)^{2}$ associated with $\tilde{\mathbf{l}}^{(2)}$, then $\mathbf{L}_{F}^{(2)}$ and $\mathbf{L}_{F}$ have the same domain, and on this domain, $\mathbf{L}_{F}^{(2)}=\mathbf{L}_{F}+S^{(2)}$.

Turning again to the operator $\mathbf{L}_{0}$ introduced in Section 1 (see (1.2)) and having domain $C_{0, \pi}^{\infty}(\Omega)^{2}$, we now present some results pertaining to this operator which were derived in [FMM]. In what follows we shall use the notation $\mathcal{D}(V)$ and $\mathcal{D}(h)$ to denote the domain of the operator $V$ and the sesquilinear form $h$ acting in some Hilbert space.

Proposition 2.4. The operator $D_{0}$ has a Friedrich's extension in $L_{2}\left(\Omega, s^{-1}\right)$, and if we denote this extension by $D$, then $D$ is a nonnegative, selfadjoint operator with domain $\mathcal{D}(D)=Z_{\pi}^{2}\left(\Omega, s^{-1}\right)$ and

$$
D u=-\frac{1+s^{2}}{s^{2}} \partial_{2}^{2} u \quad \forall u \in \mathcal{D}(D) .
$$

Furthermore, if $\mu<0$, then $(D-\mu)^{-1}$ maps $C_{0, \pi}^{\infty}$ into itself. 
PROPOSITION 2.5. The operators $B$ and $C$ are closable in $L_{2}\left(\Omega, s^{-1}\right)$ and their closures, $\bar{B}$ and $\bar{C}$, respectively, satisfy $\bar{B} \subset C^{*}$ and $\bar{C} \subset B^{*}$. Furthermore, for $\mu<0$, the operator $(D-\mu)^{-1} C$ is also closable in $L_{2}\left(\Omega, s^{-1}\right)$.

We henceforth denote the closure of $(D-\mu)^{-1} C$ by $\overline{(D-\mu)^{-1} C}$.

Let $\mu<0$. Then we also know from [FMM] that $\mathbf{L}_{0}-\mu$ admits the representation

$$
\left(\begin{array}{cc}
I & B(D-\mu)^{-1} \\
0 & I
\end{array}\right)\left(\begin{array}{cc}
T^{(0)}(\mu) & 0 \\
0 & D-\mu
\end{array}\right)\left(\begin{array}{cc}
I & 0 \\
(D-\mu)^{-1} C & I
\end{array}\right),
$$

where $I$ denotes the identity operator in $L_{2}\left(\Omega, s^{-1}\right)$ and $T^{(0)}(\mu)$ denotes the symmetric operator $(A-\mu)-B(D-\mu)^{-1} C$. Furthermore, it is shown in [FMM] that $T^{(0)}(\mu)$ has a Friedrich's extension $T(\mu)$ in $L_{2}\left(\Omega, s^{-1}\right)$ which is selfadjoint, positive, invertible, and satisfies $T(\mu) \geq-\mu$, while in addition,

$$
\mathcal{D}\left(T(\mu)^{1 / 2}\right) \subset \mathcal{D}\left(\overline{(D-\mu)^{-1} C}\right) .
$$

Note that this last result implies that

$$
G(\mu)=\overline{(D-\mu)^{-1} C} T(\mu)^{-1 / 2}
$$

is a bounded operator in $L_{2}\left(\Omega, s^{-1}\right)$. Using these facts, it is shown in [FMM] that $\mathbf{L}_{F}-\mu$ admits the representation

$$
\begin{aligned}
\mathbf{L}_{F}-\mu=\left(\begin{array}{cc}
T(\mu)^{1 / 2} & T(\mu)^{1 / 2} G(\mu)^{*}(D-\mu)^{1 / 2} \\
0 & (D-\mu)^{1 / 2}
\end{array}\right) & \\
& \times\left(\begin{array}{cc}
T(\mu)^{1 / 2} & 0 \\
(D-\mu)^{1 / 2} G(\mu) T(\mu)^{1 / 2} & (D-\mu)^{1 / 2}
\end{array}\right),
\end{aligned}
$$

and hence

$$
\left(\mathbf{L}_{F}-\mu\right)^{-1}=\left(\begin{array}{cc}
T(\mu)^{-1} & -T(\mu)^{-1 / 2} G(\mu)^{*} \\
-G(\mu) T(\mu)^{-1 / 2} & G(\mu) G(\mu)^{*}+(D-\mu)^{-1}
\end{array}\right) .
$$

\section{The main result}

In this section we use the results of Section 2 as well as those of [FMM, FM] to derive the main result of this paper, which is given in the following theorem.

THEOREM 3.1. Suppose that $d=0$ almost everywhere in $\Omega$. Then $\sigma_{\mathrm{ess}}\left(\mathbf{L}_{F}^{(2)}\right)=\sigma_{\mathrm{ess}}\left(\mathbf{L}_{F}^{(1)}\right)$, and hence

$$
\sigma_{\mathrm{ess}}\left(\mathbf{L}_{F}^{(2)}\right)= \begin{cases}\{0\} \cup \bigcup_{m=1}^{\infty}\left[m^{2}, \frac{m^{2}}{\omega^{2}}\right] & \text { if } 0<\omega \leq 1, \\ \{0\} \cup \bigcup_{m=1}^{\infty}\left[\frac{m^{2}}{\omega^{2}}, m^{2}\right] & \text { if } \omega>1 .\end{cases}
$$


Since the equality (3.1) with $\mathbf{L}_{F}^{(2)}$ replaced by $\mathbf{L}_{F}^{(1)}$ is proved in [FM], we need only prove the first assertion of the theorem. To this end some preliminary results are required. Accordingly, let us assume henceforth that $\mu<-\gamma_{0}$ and turn our attention again to the operator $T(\mu)$ introduced in Section 2. Then we observe from [FMM, Section 6] that if $u \in C_{0, \pi}^{\infty}(\Omega)$, then $T(\mu) u$ can be expanded in $L_{2}\left(\Omega, s^{-1}\right)$ by means of a Fourier series expansion with respect to the variable $t$, that is,

$$
T(\mu) u=\sum_{m=-\infty}^{\infty} T_{m}(\mu) Q_{m} u \otimes h_{m},
$$

where

$$
\begin{gathered}
h_{m}(t)=\frac{1}{\sqrt{2 \pi}} e^{i m t}, \quad\left(Q_{m} u\right)(s)=\int_{0}^{2 \pi} u(s, t) \overline{h_{m}(t)} d t, \\
T_{0}(\mu)=-\omega^{-2} s \partial_{1} \frac{1}{s} \partial_{1}-\mu, \\
T_{m}(\mu)=-\omega^{-2} s \partial_{1} \frac{m^{2}-\mu}{\left(1+s^{2}\right) m^{2}-\mu s^{2}} s \partial_{1}+\frac{m^{2}}{\omega^{2}}-\mu \quad \text { for } m \neq 0 .
\end{gathered}
$$

From $[\mathrm{FMM}]$ we have the following results.

Proposition 3.2. $T_{0}(\mu)$, with domain $C_{0}^{\infty}((0,1))$, has a Friedrich's extension $T_{0, F}(\mu)$ in $L_{2}\left((0,1), s^{-1}\right)$ which has the following properties.

(1) $T_{0, F}(\mu)=-\omega^{-2} s \partial_{1} 1 / s \partial_{1}-\mu$ and its domain is

$$
\left\{f \in H^{1}\left((0,1), s^{-1}\right) \mid s \partial_{1} s^{-1} \partial_{1} f \in L_{2}\left((0,1), s^{-1}\right), f(1)=0\right\} .
$$

(2) $T_{0, F}(\mu)$ is a selfadjoint, strictly positive operator in $L_{2}\left((0,1), s^{-1}\right)$ with compact inverse.

(3) $T_{0, F}(\mu)$ is precisely the selfadjoint operator in $L_{2}\left((0,1), s^{-1}\right)$ that is associated with the closed, densely defined, nonnegative form

$$
h(\cdot, \cdot)=\left(\overline{S_{0}(\mu)} \cdot \overline{S_{0}(\mu)} \cdot\right)_{0,(0,1), s^{-1}}-\mu(\cdot, \cdot)_{0,(0,1), s^{-1}}
$$

and

$$
T_{0, F}(\mu)=S_{0}(\mu)^{*} \overline{S_{0}(\mu)}-\mu,
$$

where

$$
\begin{gathered}
\mathcal{D}\left(\overline{S_{0}(\mu)}\right)=\left\{f \in H^{1}\left((0,1), s^{-1}\right) \mid f(1)=0\right\}, \\
\mathcal{D}\left(S_{0}(\mu)^{*}\right)=\left\{f \in L_{2}\left((0,1), s^{-1}\right) \mid s \partial_{1} s^{-1} f \in L_{2}\left((0,1) s^{-1}\right)\right\},
\end{gathered}
$$

and $\overline{S_{0}(\mu)}=\omega^{-1} \partial_{1}$ and $S_{0}(\mu)^{*}=-\omega^{-1} s \partial_{1} s^{-1}$ on their respective domains.

Proposition 3.3. Suppose that $m \neq 0$. Then $T_{m}(\mu)$, with domain $C_{0}^{\infty}((0,1))$, has a Friedrich's extension $T_{m, F}(\mu)$ in $L_{2}\left((0,1), s^{-1}\right)$, which has the following properties. 
(1)

$$
T_{m, F}(\mu)=-\omega^{-2} s \partial_{1} \frac{m^{2}-\mu}{\left(1+s^{2}\right) m^{2}-\mu s^{2}} s \partial_{1}+\frac{m^{2}}{\omega^{2}}-\mu
$$

and

$$
\begin{gathered}
\mathcal{D}\left(T_{m, F}(\mu)\right)=\left\{f \in L_{2}\left((0,1), s^{-1}\right) \mid\left(s \partial_{1}\right)^{j} f \in L_{2}\left((0,1), s^{-1}\right)\right. \\
\text { for } j=1,2, f(1)=0\} .
\end{gathered}
$$

(2) $T_{m, F}(\mu)$ is a selfadjoint and strictly positive operator in $L_{2}\left((0,1), s^{-1}\right)$.

(3) $T_{m, F}(\mu)$ is precisely the selfadjoint operator in $L_{2}\left((0,1), s^{-1}\right)$ that is associated with the closed, densely defined, nonnegative form

$$
h(\cdot, \cdot)=\left(\overline{S_{m}(\mu)} \cdot, \overline{S_{m}(\mu)} \cdot\right)_{0,(0,1), s^{-1}}+\left(\frac{m^{2}}{\omega^{2}}-\mu\right)(\cdot, \cdot)_{0,(0,1), s^{-1}}
$$

and

$$
T_{m, F}(\mu)=S_{m}(\mu)^{*} \overline{S_{m}(\mu)}+\frac{m^{2}}{\omega^{2}}-\mu,
$$

where

$$
\begin{gathered}
\mathcal{D}\left(\overline{S_{m}(\mu)}\right)=\left\{f \in L_{2}\left((0,1), s^{-1}\right) \mid s \partial_{1} f \in L_{2}\left((0,1), s^{-1}\right), f(1)=0\right\}, \\
\mathcal{D}\left(S_{m}(\mu)^{*}\right)=\left\{L_{2}\left((0,1), s^{-1}\right) \mid s \partial_{1} f \in L_{2}\left((0,1), s^{-1}\right)\right\}
\end{gathered}
$$

and

$$
\begin{aligned}
\overline{S_{m}(\mu)} & =\frac{s}{\omega} \sqrt{\frac{m^{2}-\mu}{\left(1+s^{2}\right) m^{2}-\mu s^{2}}} \partial_{1}, \\
S_{m}(\mu)^{*} & =-s \omega^{-1} \partial_{1} \sqrt{\frac{m^{2}-\mu}{\left(1+s^{2}\right) m^{2}-\mu s^{2}}}
\end{aligned}
$$

on their respective domains.

Proposition 3.4. The domain $\mathcal{D}(T(\mu))$ of $T(\mu)$ is the set of all $f \in L_{2}\left(\Omega, s^{-1}\right)$ such that $Q_{m} f \in \mathcal{D}\left(T_{m, F}(\mu)\right)$ for all $m \in \mathbb{Z}$ and

$$
\sum_{m=-\infty}^{\infty}\left\|T_{m, F}(\mu) Q_{m} f\right\|_{0,(0,1), s^{-1}}^{2}<\infty .
$$

Furthermore, for all $f \in \mathcal{D}(T(\mu))$,

$$
T(\mu) f=\sum_{m=-\infty}^{\infty}\left(T_{m, F}(\mu) Q_{m} f\right) \otimes h_{m} .
$$


We now use the results just cited as well as those of Section 2 to derive some results which will be used in the proof of Theorem 3.1. To this end let $S_{0}^{(2)}=S^{(2)}-S^{(1)}$. Then we observe that

$$
\mathbf{L}_{F}^{(2)}-\mu=\mathbf{L}_{F}-\mu+S^{2}=\mathbf{L}_{F}^{(1)}-\mu+S_{0}^{(2)}
$$

and that

$$
\left\|S_{0}^{(2)}\left(\mathbf{L}_{F}^{(1)}-\mu\right)^{-1}\right\|_{L_{2}\left(\Omega, s^{-1}\right)^{2} \rightarrow L_{2}\left(\Omega, s^{-1}\right)^{2}}<1,
$$

that

$$
\left(\mathbf{L}_{F}^{(2)}-\mu\right)^{-1}=\left(\mathbf{L}_{F}^{(1)}-\mu\right)^{-1}\left(I+S_{0}^{(2)}\left(\mathbf{L}_{F}^{(1)}-\mu\right)^{-1}\right)^{-1},
$$

and hence it follows that

$$
\begin{aligned}
\left(\mathbf{L}_{F}^{(2)}\right. & -\mu)^{-1}-\left(\mathbf{L}_{F}^{(1)}-\mu\right)^{-1} \\
& =-\left(\mathbf{L}_{F}^{(1)}-\mu\right)^{-1} S_{0}^{(2)}\left(\mathbf{L}_{F}^{(1)}-\mu\right)^{-1}\left(I+S_{0}^{(2)}\left(\mathbf{L}_{F}^{(1)}-\mu\right)^{-1}\right)^{-1} .
\end{aligned}
$$

Our goal now is to prove that $\left(\mathbf{L}_{F}^{(2)}-\mu\right)^{-1}-\left(\mathbf{L}_{F}^{(1)}-\mu\right)^{-1}$ is a compact operator in $L_{2}\left(\Omega, s^{-1}\right)^{2}$ and then use this fact to prove Theorem 3.1. Then since

$$
\left(\mathbf{L}_{F}^{(1)}-\mu\right)^{-1}=\left(\mathbf{L}_{F}^{(1)}-\mu\right)^{-1}-\left(\mathbf{L}_{F}-\mu\right)^{-1}+\left(\mathbf{L}_{F}-\mu\right)^{-1},
$$

and since we know from $[\mathrm{FM}]$ that $\left(\mathbf{L}_{F}^{(1)}-\mu\right)^{-1}-\left(\mathbf{L}_{F}-\mu\right)^{-1}$ is a compact operator in $L_{2}\left(\Omega, s^{-1}\right)^{2}$, we see that our goal will be achieved if we can show that

$$
X(\mu)=\left(\mathbf{L}_{F}-\mu\right)^{-1} S_{0}^{(2)}\left(\mathbf{L}_{F}-\mu\right)^{-1}
$$

is a compact operator in $L_{2}\left(\Omega, s^{-1}\right)^{2}$.

Accordingly, let $X_{j, k}(\mu)$, where $1 \leq j, k \leq 2$, denote the components of $X(\mu)$. Then it follows from (2.3) that

$$
\begin{aligned}
X_{11}(\mu)=- & T(\mu)^{-1 / 2} G(\mu)^{*} S_{c} T(\mu)^{-1} \\
& -\left(T(\mu)^{-1} S_{b}-T(\mu)^{-1 / 2} G(\mu)^{*} S_{d}\right) G(\mu) T(\mu)^{-1 / 2}, \\
X_{12}(\mu)=T(\mu)^{-1 / 2} G(\mu)^{*} S_{c} T(\mu)^{-1 / 2} G(\mu)^{*} & \quad\left(T(\mu)^{-1} S_{b}-T(\mu)^{-1 / 2} G(\mu)^{*} S_{d}\right)\left(G(\mu) G(\mu)^{*}+(D-\mu)^{-1}\right), \\
& \left.\quad+(\mu) G(\mu)^{*}+(D-\mu)^{-1}\right) S_{c} T(\mu)^{-1} \\
X_{21}(\mu)=( & \quad\left(G(\mu) T(\mu)^{-1 / 2} S_{b}-\left(G(\mu) G(\mu)^{*}+(D-\mu)^{-1}\right) S_{d}\right) G T(\mu)^{-1 / 2}, \\
& \quad\left(G(\mu) G(\mu)^{*}+(D-\mu)^{-1}\right) S_{c} T(\mu)^{-1 / 2} G(\mu)^{*} \\
X_{22}(\mu)=- & \quad\left(G(\mu) T(\mu)^{-1 / 2} S_{b}-\left(G(\mu) G(\mu)^{*}+(D-\mu)^{-1}\right) S_{d}\right) \\
& \quad \times\left(G(\mu) G(\mu)^{*}+(D-\mu)^{-1}\right) .
\end{aligned}
$$

Proposition 3.5. $S_{c} T(\mu)^{-1}, S_{c} T(\mu)^{-1 / 2}$, and $S_{d} G(\mu) T(\mu)^{-1 / 2}$ are compact operators in $L_{2}\left(\Omega, s^{-1}\right)$. 
Proof. Since

$$
\left\|S_{c} T(\mu)^{-1} f\right\|_{0, \Omega, s^{-1}}^{2}=\left(T(\mu)^{-1} S_{|c|^{2}} T(\mu)^{-1} f, f\right) \quad \forall f \in L_{2}\left(\Omega, s^{-1}\right)
$$

and since we know from [FM] that $T(\mu)^{-1} S_{|c|^{2}} T(\mu)^{-1}$ is a compact operator in $L_{2}\left(\Omega, s^{-1}\right)$, it follows that this last result is also true for $S_{c} T(\mu)^{-1}$. Furthermore, since

$$
\left\|S_{c} T(\mu)^{-1 / 2} f\right\|_{0, \Omega, s^{-1}}^{2}=(V(\mu) f, f)_{0, \Omega, s^{-1}},
$$

where $V(\mu)=T(\mu)^{-1 / 2} S_{|c|^{2}} T(\mu)^{-1 / 2}$, and since we know from [FM] that $V(\mu)$ is a compact operator in $L_{2}\left(\Omega, s^{-1}\right)$, it follows that this last result is also true for $S_{c} T(\mu)^{-1 / 2}$.

Turning next to the assertion concerning $S_{d} G(\mu) T(\mu)^{-1 / 2}$, let us observe from [FMM] that

$$
G(\mu) T(\mu)^{-1 / 2} f=\sum_{\substack{m=-\infty \\ m \neq 0}}^{\infty} G_{m}(\mu) T_{m, F}(\mu)^{-1 / 2} Q_{m} f \otimes h_{m}
$$

for all $f \in L_{2}\left(\Omega, s^{-1}\right)$, where

$$
G_{m}(\mu)=-\frac{i m}{\sqrt{\left(m^{2}-\mu\right)\left[\left(1+s^{2}\right) m^{2}-\mu s^{2}\right]}} \overline{S_{m}(\mu)} T_{m, F}(\mu)^{-1 / 2} .
$$

We note from part (3) of Proposition 3.3 that

$$
\mathcal{D}\left(T_{m, F}(\mu)^{1 / 2}\right)=\mathcal{D}\left(\overline{S_{m}(\mu)}\right),
$$

and hence it follows that $\overline{S_{m}(\mu)} T_{m, F}(\mu)^{-1 / 2}$ is a bounded operator in $L_{2}\left((0,1), s^{-1}\right)$. Furthermore, since

$$
\begin{aligned}
& \|f\|_{0,(0,1), s^{-1}}^{2} \\
& \quad=\left\|\overline{S_{m}(\mu)} T_{m, F}(\mu)^{-1 / 2} f\right\|_{0,(0,1), s^{-1}}^{2}+\left(\frac{m^{2}}{\omega^{2}}-\mu\right)\left\|T_{m, F}(\mu)^{-1 / 2} f\right\|_{0,(0,1), s^{-1}}^{2}
\end{aligned}
$$

for all $f \in L_{2}\left((0,1), s^{-1}\right)$, it also follows that

$$
\left\|G_{m}(\mu)\right\|_{L_{2}\left((0,1), s^{-1}\right) \rightarrow L_{2}\left((0,1), s^{-1}\right)} \leq \frac{1}{|m|}
$$

and

$$
\left\|T_{m, F}(\mu)^{-1 / 2}\right\|_{L_{2}\left((0,1), s^{-1}\right) \rightarrow L_{2}\left((0,1), s^{-1}\right)} \leq \frac{\omega}{\sqrt{m^{2}-\omega^{2} \mu}} .
$$

Lastly we observe from (3.2) that

$$
G_{m}(\mu) T_{m, F}(\mu)^{-1 / 2}=-\frac{i m}{\sqrt{\left(m^{2}-\mu\right)\left[\left(1+s^{2}\right) m^{2}-\mu s^{2}\right]}} \overline{S_{m}(\mu)} T_{m, F}(\mu)^{-1},
$$


from which it follows that $G_{m}(\mu) T_{m, F}(\mu)^{-1 / 2}$ is a bounded linear transformation from $L_{2}\left((0,1), s^{-1}\right)$ into $L_{2}((\delta, 1))$ whose range lies in $H^{1}((\delta, 1))$ for any $\delta$ satisfying $0<\delta<1$. Hence we conclude from the closed graph theorem that the mapping

$$
G_{m}(\mu) T_{m, F}(\mu)^{-1 / 2}: L_{2}\left((0,1), s^{-1}\right) \rightarrow H^{1}((\delta, 1))
$$

is bounded. Let us now use these facts to prove the assertion concerning the compactness of $S_{d} G(\mu) T(\mu)^{-1 / 2}$; since

$$
\left\|S_{d} G(\mu) T(\mu)^{-1 / 2} f\right\|_{0, \Omega, s^{-1}}^{2}=\left(T(\mu)^{-1 / 2} G(\mu)^{*} S_{d^{2}} G(\mu) T(\mu)^{-1 / 2} f, f\right)_{0, \Omega, s^{-1}},
$$

we see that the assertion will be proved if we can show that

$$
T(\mu)^{-1 / 2} G(\mu)^{*} S_{d^{2}} G T(\mu)^{-1 / 2}
$$

is a compact operator in $L_{2}\left(\Omega, s^{-1}\right)$.

To this end let

$$
d_{r}=d_{r}(s)=(2 \pi)^{-1 / 2}\left(Q_{r} d^{2}\right)(s) \quad \forall r \in \mathbb{Z} .
$$

Then it follows from the foregoing results that for all $f \in L_{2}\left(\Omega, s^{-1}\right)$,

$$
\begin{aligned}
& \left\|T(\mu)^{-1 / 2} G(\mu)^{*} S_{d^{2}} G(\mu) T(\mu)^{-1 / 2} f\right\|_{0, \Omega, s^{-1}} \\
& =\left(\sum_{m=-\infty}^{\infty} \| \sum_{r=-\infty}^{\infty} T_{m, F}(\mu)^{-1 / 2} G_{m}(\mu)^{*} d_{r} G_{m-r}(\mu)\right. \\
& \left.\quad \times T_{m-r, F}(\mu)^{-1 / 2} Q_{m-r} f \|_{0,(0,1), s^{-1}}^{2}\right)^{1 / 2} \\
& \leq \sum_{r=-\infty}^{\infty} \frac{1}{1+r^{2}}\left(\sum_{m=-\infty}^{\infty} \| T_{m, F}(\mu)^{-1 / 2} G_{m}(\mu)^{*} \tilde{d}_{r} G_{m-r}(\mu)\right. \\
& \quad \times T_{m-r, F}(\mu)^{-1 / 2} Q_{m-r} f \|_{0, \Omega, s^{-1}}^{2},
\end{aligned}
$$

where $\tilde{d}_{r}=\left(1+r^{2}\right) d_{r}$, and $G_{0}(\mu)$ is defined to be 0 , and where we have made use of Minkowski's inequality. Note also that

$$
\tilde{d}_{r}^{2} \leq \frac{1}{\pi} \int_{0}^{2 \pi}\left(d^{2}(s, \cdot)^{2}+\left|\partial_{2}^{2} d^{2}(s, \cdot)\right|^{2}\right) d t \leq 2 \tilde{\gamma}_{d}^{2},
$$

where $\tilde{\gamma}_{d}^{2}=\gamma_{d}^{4}+\left\|\partial_{2}^{2} d^{2}\right\|_{L_{\infty}(\Omega)}^{2}$.

Next let $\mathcal{F}$ denote a bounded set in $L_{2}\left(\Omega, s^{-1}\right)$. Then it follows from the fact that $\left\|Q_{r} f\right\|_{0,(0,1), s^{-1}} \leq\|f\|_{0, \Omega, s^{-1}}$ for all $f \in L_{2}\left(\Omega, s^{-1}\right)$ that $\left\{Q_{r} f \mid r \in \mathbb{Z}, f \in \mathcal{F}\right\}$ is a bounded set in $L_{2}\left((0,1), s^{-1}\right)$. Hence if we fix an integer $\ell \geq 2$ and observe from 
Propositions 3.2, 3.3, and the text following (3.2) that for each pair $(m, r)$, in $\mathbb{Z}^{2}$ with $m \neq r$, the set $\left\{G_{m-r}(\mu) T_{m-r, F}(\mu)^{-1 / 2} Q_{m-r} f\right\}_{f \in \mathcal{F}}$ is bounded in $H^{1}\left(\left(\ell^{-1}, 1\right)\right)$, then we can appeal firstly to Rellich's theorem and then to a standard diagonalization procedure to select a sequence of vectors $\left\{f_{n}\right\}_{1}^{\infty}$ in $\mathcal{F}$ such that the sequence

$$
\left\{G_{m-r}(\mu) T_{m-r, F}(\mu)^{-1 / 2} Q_{m-r} f_{n}\right\}_{n=1}^{\infty}
$$

converges in $L_{2}\left(\left(\ell^{-1}, 1\right)\right)$ for every pair $(m, r) \in \mathbb{Z}^{2}$. Since this result is true for every $\ell \geq 2$, we can again appeal to a standard diagonalization procedure to choose the sequence $\left\{f_{n}\right\}_{1}^{\infty}$ just cited so that the sequence

$$
\left\{G_{m-r}(\mu) T_{m-r, F}(\mu)^{-1 / 2} Q_{m-r} f_{n}\right\}_{n=1}^{\infty}
$$

converges in $L_{2}\left(\left(\ell^{-1}, 1\right)\right)$ for every pair $(m, r) \in \mathbb{Z}^{2}$ and for every integer $\ell \geq 2$.

Let $\ell, m_{0}$, and $r_{0}$ denote integers at least 2 with $m_{0}>r_{0}$. Then with $\left\{f_{n}\right\}_{1}^{\infty}$ denoting the sequence in $\mathcal{F}$ defined above, it follows from (3.3) that for $j, k \geq 1$,

$$
\left\|T(\mu)^{-1 / 2} G(\mu)^{*} S_{d^{2}} G(\mu) T(\mu)^{-1 / 2}\left(f_{j}-f_{k}\right)\right\|_{0, \Omega, s^{-1}} \leq \sum_{j=0}^{3} J_{i},
$$

where

$$
\begin{gathered}
J_{0}=\sum_{r=-\infty}^{\infty} \frac{1}{1+r^{2}}\left(\sum_{\substack{m=-\infty \\
m \neq 0}}^{\infty}\left\|V_{m, r}(\mu)\left(f_{j}-f_{k}\right)\right\|_{0,\left(0, \ell^{-1}\right), s^{-1}}^{2}\right)^{1 / 2}, \\
J_{1}=\sum_{|r|>r_{0}} \frac{1}{1+r^{2}}\left(\sum_{\substack{m=-\infty \\
m \neq 0}}^{\infty}\left\|V_{m, r}(\mu)\left(f_{j}-f_{k}\right)\right\|_{0,\left(\ell^{-1}, 1\right), s^{-1}}^{2}\right)^{1 / 2}, \\
J_{2}=\sum_{|r| \leq r_{0}} \frac{1}{1+r^{2}}\left(\sum_{\substack{|m|>m_{0} \\
J_{3}}}=\sum_{|r| \leq r_{0}} \frac{1}{1+r^{2}}\left(\sum_{\substack{|m| \leq m_{0} \\
m \neq 0}}\left\|V_{m, r}(\mu)\left(f_{j}-f_{k}\right)\right\|_{0,\left(\ell^{-1}, 1\right), s^{-1}}^{2}\right)^{1 / 2},\right. \\
V_{m, r}(\mu)=\frac{\left.\left.f_{j}-f_{k}\right) \|_{0,\left(\ell^{-1}, 1\right), s^{-1}}^{2}\right)^{1 / 2},}{\sqrt{m^{2}\left(m^{2}-\mu\right)}} \tilde{d}_{r} G_{m-r}(\mu) T_{m-r, F}(\mu)^{-1 / 2} Q_{m-r} .
\end{gathered}
$$

It follows from the results above that

$$
\begin{gathered}
J_{0} \leq C_{0} \rho_{d^{2}}\left(\ell^{-1}\right)\left(\sum_{r=-\infty}^{\infty} \frac{1}{1+r^{2}}\right)\left(\sum_{\substack{m=-\infty \\
m \neq 0}}^{\infty} \frac{1}{m^{2}\left(m^{2}-\mu\right)}\right)^{1 / 2}, \\
J_{1} \leq C_{0} \tilde{\gamma}_{d}\left(\sum_{r>r_{0}} \frac{1}{1+r^{2}}\right)\left(\sum_{\substack{m=-\infty \\
m \neq 0}}^{\infty} \frac{1}{m^{2}\left(m^{2}-\mu\right)}\right)^{1 / 2},
\end{gathered}
$$


and

$$
\begin{aligned}
J_{2} \leq C_{0} \tilde{\gamma}_{d}\left(\sum_{|r| \leq r_{0}} \frac{1}{1+r^{2}}\right) \\
\quad \times\left(\sum_{|m|>m_{0}} \frac{1}{m^{2}\left(m^{2}-\mu\right)\left(m-r_{0}\right)^{2}\left[\left(m-r_{0}\right)^{2}-\mu\right]}\right)^{1 / 2}, \\
J_{3} \leq C_{0} \tilde{\gamma}_{d}\left(\sum_{|r| \leq r_{0}} \frac{1}{1+r^{2}}\right) \\
\quad \times\left(\sum_{\substack{|m| \leq m_{0} \\
m \neq 0}}\left\|G_{m-r}(\mu) T_{m-r, F}(\mu)^{-1 / 2} Q_{m-r}\left(f_{j}-f_{k}\right)\right\|_{0,\left(\ell^{-1}, 1\right), s^{-1}}^{2}\right)^{1 / 2},
\end{aligned}
$$

where

$$
C_{0}=2 \sqrt{2}(1+M)\left(1+\omega^{2}\right) \quad \text { and } \quad M=\sup _{f \in \mathcal{F}}\|f\|_{0, \Omega, s^{-1}} .
$$

Hence given any $\epsilon>0$, we can choose $\ell$ so large that $J_{0}<\epsilon / 4$, then choose $r_{0}$ so large that $J_{1}<\epsilon / 4$, and finally choose $m_{0}$ large enough that $J_{2}<\epsilon / 4$. Then with $\ell, r_{0}$, and $m_{0}$ so chosen we can choose $n_{0}$ large enough that $J_{3}<\epsilon / 4$ for $j, k \geq n_{0}$. Thus we have shown that there exists an integer $n_{0}$ such that

$$
\left\|T(\mu)^{-1 / 2} G(\mu)^{*} S_{d^{2}} G(\mu) T(\mu)^{-1 / 2}\left(f_{j}-f_{k}\right)\right\|_{0, \Omega, s^{-1}}<\epsilon \quad \forall j, k \geq n_{0},
$$

and since $\epsilon$ is arbitrary, we conclude that

$$
T(\mu)^{-1 / 2} G(\mu)^{*} S_{d^{2}} G(\mu) T(\mu)^{-1 / 2}
$$

is a compact operator in $L_{2}\left(\Omega, s^{-1}\right)$, and this completes the proof of the proposition.

Proof of TheOrem 3.1. It follows from Proposition 3.5 and from a consideration of adjoints that $X_{11}(\mu), X_{12}(\mu), X_{21}(\mu)$, and

$$
X_{22}(\mu)-\left(G(\mu) G(\mu)^{*}+(D-\mu)^{-1}\right) S_{d}\left(G(\mu) G(\mu)^{*}+(D-\mu)^{-1}\right)
$$

are compact operators in $L_{2}\left(\Omega, s^{-1}\right)$. Thus if we suppose that $\|d\|_{L_{\infty}(\Omega)}=0$, then $X(\mu)$, and hence also $\left(L_{F}^{(2)}-\mu\right)^{-1}-\left(L_{F}^{(1)}-\mu\right)^{-1}$, are compact operators in $L_{2}\left(\Omega, s^{-1}\right)^{2}$; and this implies that $\sigma_{\mathrm{ess}}\left(L_{F}^{(2)}-\mu\right)^{-1}=\sigma_{\mathrm{ess}}\left(L_{F}^{(1)}-\mu\right)^{-1}$. It is an immediate consequence of this result that $\sigma_{\mathrm{ess}}\left(L_{F}^{(2)}\right)=\sigma_{\mathrm{ess}}\left(L_{F}^{(1)}\right)$.

\section{References}

[DG1] J. Descloux and G. Geymonat, 'On the essential spectrum of an operator relative to the stability of a plasma in toroidal geometry', École Polytechnique Fédérale de Lausanne (1979). 
[DG2] J. Descloux and G. Geymonat, 'Sur le spectre essentiel d'un opérateur relatif à la stabilité d'un plasma en géométrie toroïdale', C. R. Acad. Sci. Paris, Sér. A 290 (1980), 795-797.

[FM] M. Faierman and R. Mennicken, 'The essential spectrum of an operator arising in twodimensional magnetohydrodynamics', Math. Nachr. 282 (2009), 422-429.

[FMM] M. Faierman, R. Mennicken and M. Möller, 'The essential spectrum of a model problem in two-dimensional magnetohydrodynamics: a proof of a conjecture of J. Descloux and G. Geymonat', Math. Nachr. 269-270 (2004), 129-149.

[GK] I. Gohberg and M. G. Krein, 'Systems of integral equations on a half line with kernels depending on the difference of arguments', Amer. Math. Soc. Transl. (2) 14 (1960), 217-287.

[K] T. Kato, Perturbation Theory for Linear Operators (Springer, New York, 1976).

M. FAIERMAN, School of Mathematics and Statistics, University of New South Wales, UNSW Sydney NSW 2052, Australia e-mail: m.faierman@unsw.edu.au

R. MENNICKEN, Department of Mathematics, University of Regensburg, D-93040 Regensburg, Germany

e-mail: reinhard.mennicken@mathematik.uni-regensburg.de 